\title{
Indian perspective about COVID-19 vaccine: an elixir bringing ray of hope to the dark pandemic
}

Sir,

India has finally begun its colossal coronavirus vaccine drive, desperately hoping to end a deadly pandemic that has killed around 1.5 lakh of its population. With an aim to vaccinate about 300 million people across the subcontinent by July, India has a huge task ahead. This large number roughly amounts to almost the entire US population.

Back in April and May 2020, with lockdown tolling across the country, it felt like losing a battle. Elective medical services were totally shut down. Only emergencies and COVID services remained operational, that too, at the risk of doctors' lives. Every single moment, we experienced the awe of acquiring infections while on duty. But hats off to the courage with which we all fought. Today medical fraternity is finding a ray of hope and feeling a great satisfaction after the vaccine has flooded the markets.

A second hit of infections with a mutated strain is threatening to derail the economies as countries have gradually begun to ease the restrictions and open up frontiers. We still need to take precautions against this new virus. In an impoverished nation with the world's worst funded healthcare system, it is still a daunting undertaking to launch the vaccine in such a massive scale. No matter baseless rumors and a torrent of hoaxes are hovering across the sky, but we need to dilute this skepticism about the vaccine and encourage the public to rush to get the COVID-19 vaccine.

Rapid community spread owing to sustained surface viability and high virulence of the virus had caused a potential threat to the community during 2020. India being the second most populous country in the world, harbored the likelihood of infecting the highest number of individuals. We witnessed around 36,000 new cases daily which was the highest in the world! $!^{1}$ The magnitude of the pandemic has already caused an overwhelming strain upon the healthcare system of the country due to number of patients exceeding the capacity of hospital beds.

The world has gone into a state of hibernation which in turn has deeply affected the economy and healthcare. Recently the World Health Organization (WHO) has reported that shortage in medicine, diagnostics and technologies has affected even cancer treatment protocols in many countries. ${ }^{2}$ When the primary approach of the public health system is to promote social distancing, personal hygiene and resource optimization by home quarantine due to lack of hospital beds everywhere, is it plausible to fill the air with nonsense rumors about the hazards reported after vaccination? Even experts are not quite sure whether these few minor side effects are secondary to vaccine administration or not.

Although there is no proven data on pregnancy complications by COVID-19 infection, yet we cannot underestimate the previously established data of increased incidences of spontaneous miscarriage, fetal growth restriction and preterm delivery in women with SARS coronavirus infection in pregnancy. ${ }^{3}$ We cannot ignore the recent report from the Centers for Disease Control and Prevention (CDC) which showed that pregnant women with COVID-19 appeared to be at higher risk of intensive care unit (ICU) admission and mechanical ventilation compared to non-pregnant peers. So, till date, no guidelines could be formulated whether pregnant females can be subjected to COVID-19 vaccination. No proper consensus can be proposed until large scale data is

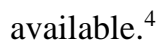

A few constructive suggestions can chart the path ahead: all reproductive medicine staff across the country must endeavor to provide a meticulous emotional and psychological proactive counselling towards the citizens: tutorials must be implemented on recognition of symptoms of COVID-19 and routes of spread, methods to curtail exposure, necessity of social distancing and reporting to health-care centers when symptoms arise; patient education about safety and efficacy of vaccination is the need of the hour; telemedicine facilities can be judiciously utilized to start proactive counselling for vaccine administration and follow-up; and toll-free helpline services must be operational $24 \times 7$ to identify problems or provide instant help to people after vaccination.

It is not clear how long the vaccine will remain effective in the body, whether it will prevent people from getting infections in future and whether the virus will mutate enough over time to require a series of new vaccines, as was in the case of influenza. Still, we look towards a brighter horizon and hope to lessen the surge of incidence after the vaccination.

Ultimately, leaving aside the debate about who gets the prime credit, we all want a safe and effective vaccine that will allow life to return to normal. It is, after all, a glimmer 
of hope for country like India with maximum number of infected individuals.

\section{Vivek Thakur', Vidhi Vanya ${ }^{2}$, Avir Sarkar ${ }^{2 *}$, Basanti Mazumdar}

${ }^{1}$ Department of Obstetrics and Gynecology, PGIMER,

Chandigarh, India

${ }^{2}$ Department of Obstetrics and Gynecology, ESIC

Medical College, Faridabad, Haryana, India

${ }^{3}$ Department of General Surgery, IMS, BHU, Varanasi,

Uttar Pradesh, India

*Correspondence to

Dr. Avir Sarkar,

E-mail: avirsarkar93@gmail.com
Organization. Available at: https://www.who.int/ news/item/01-06-2020-covid-19-significantlyimpacts-health-services-for-noncommunicablediseases. Accessed on: 04 April 2021.

3. Wong SF, Chow KM, Leung TN. Pregnancy and perinatal outcomes of women with severe acute respiratory syndrome. Am J Obstet Gynecol. 2004;191(2):292-7.

4. Centres for Disease Control and Prevention. Data on COVID-19 during pregnancy. Available at: https://www.cdc.gov/coronavirus/2019-ncov/casesupdates/special-populations/pregnancy-data-oncovid-19/what-cdc-is-doing.html. Accessed on: 04 April 2021.

\section{REFERENCES}

1. WHO Coronavirus Disease (COVID-19) Dashboard. Available at: https://covid19.who.int/. Accessed on: 04 April 2021.

2. COVID-19 significantly impacts health services for non-communicable diseases [Internet]. World Health

Cite this article as: Thakur V, Vanya V, Sarkar A, Mazumdar B. Indian perspective about COVID-19 vaccine: an elixir bringing ray of hope to the dark pandemic. Int J Reprod Contracept Obstet Gynecol 2021;10:2136-7. 